\title{
QUANTUM JACOBI FORMS AND FINITE EVALUATIONS OF UNIMODAL RANK GENERATING FUNCTIONS
}

\author{
KATHRIN BRINGMANN AND AMANDA FOLSOM
}

In honor of Ernst-Ulrich Gekeler

\begin{abstract}
In this paper, we introduce the notion of a quantum Jacobi form, and offer the twovariable combinatorial generating function for ranks of strongly unimodal sequences as an example. We then use its quantum Jacobi properties to establish a new, simpler expression for this function as a two-variable Laurent polynomial when evaluated at pairs of rational numbers. Our results also yield a new expression for radial limits associated to the partition rank and crank functions previously studied by Ono, Rhoades, and the second author.
\end{abstract}

\section{IntroduCtions AND STATEMENT OF RESUlts}

A sequence of integers $\left\{a_{j}\right\}_{j=1}^{s}(s \in \mathbb{N})$ is called a strongly unimodal sequence of size $n$ if there exists a positive integer $k$ such that

$$
0<a_{1}<a_{2}<\ldots<a_{k}>a_{k+1}>\ldots>a_{s}>0
$$

and $a_{1}+\cdots+a_{s}=n$. The rank of a strongly unimodal sequence is equal to $s-2 k+1$, the number of terms after the maximal term minus the number of terms that precede it. Let $u(m, n)$ denote the number of such size $n$ and rank $m$ sequences. The two-variable generating function $U(w ; q)$ for ranks of strongly unimodal sequences can be expressed as a $q$-hypergeometric series as follows

$$
U(w ; q):=\sum_{n=1}^{\infty} \sum_{m=-\infty}^{\infty} u(m, n)(-w)^{m} q^{n}=\sum_{n=0}^{\infty}(w q ; q)_{n}\left(w^{-1} q ; q\right)_{n} q^{n+1},
$$

where for $n \in \mathbb{N}_{0} \cup\{\infty\}$, the $q$-Pochhammer symbol is defined by $(w ; q)_{n}:=\prod_{j=0}^{n-1}\left(1-w q^{j}\right)$.

Various specializations in the variable $w$ of this function have been of interest. Namely, if $w=1$, $U(1 ; q)$ is related to a function originally studied by Kontsevich, later by Zagier, and also in [4], which we recall in Section 2. In [4], $U(1 ; q)$ is also written as a Hecke-type sum, which yields congruences for its coefficients. Hecke-type sums for the general function $U(w ; q)$ may be found in [9]. In the special case $w= \pm i, U( \pm i ; q)$ is the 3rd order mock theta function of Ramanujan

$$
U( \pm i ; q)=\Psi(q):=\sum_{n=1}^{\infty} \frac{q^{n^{2}}}{\left(q ; q^{2}\right)_{n}} .
$$

Specializing to $w=-1$ gives

$$
U(q):=U(-1 ; q)=: \sum_{n=0}^{\infty} u(n) q^{n}
$$

2010 Mathematics Subject Classification. 05A17, 11F03, 11F30, 11F50, 11P55, 11P82.

The research of the first author was supported by the Alfried Krupp Prize for Young University Teachers of the Krupp foundation and the research leading to these results has received funding from the European Research Council under the European Union's Seventh Framework Programme (FP/2007-2013) / ERC Grant agreement n. 335220 - AQSER. The second author is grateful for the support of NSF CAREER grant DMS-1449679, and for the hospitality provided by the Max Planck Institute for Mathematics, Bonn, and the Institute for Advanced Study, Princeton, under NSF grant DMS-1128155. 
the generating function for $u(n)$, the number of strongly unimodal sequences of $n$. Rhoades [11] showed that $U(q)$ is a mixed mock modular form (i.e., a linear combination of modular and mock modular forms) and studied the asymptotic behavior of $u(n)$, as $n \rightarrow \infty$. In general, $U(w ; q)$ also nicely fits into a modular framework. To give the precise statement, let $a \in \mathbb{Z}$ and $b \in \mathbb{N}$ with $\operatorname{gcd}(a, b)=1$, and set $\zeta_{N}:=e^{2 \pi i / N}$. Fix $w=\zeta_{b}^{a}$ to be a root of unity, and consider the function $\phi_{a / b}(x):=U\left(\zeta_{b}^{a} ; e^{2 \pi i x}\right)$. It is now known $[4,5]$ that the functions $\phi_{a / b}$ are essentially (up to multiplication by $q$-powers) quantum modular forms. Quantum modular forms (see Section 2.2), recently defined by Zagier, are complex valued functions whose domains are subsets of $\mathbb{Q}$, and which satisfy modular transformation properties there, up to the addition of an error term which extends to a suitably analytic or continuous function in $\mathbb{R}$.

As a two-variable function on $\mathbb{C} \times \mathbb{H}$, the function

$$
Y^{+}(z ; \tau):=-2 i \sin (\pi z) q^{-\frac{1}{24}} \mathcal{U}(z ; \tau),
$$

where $\mathcal{U}\left(w_{1} ; w_{2}\right):=U\left(e\left(w_{1}\right) ; e\left(w_{2}\right)\right)$, and $e(\alpha):=e^{2 \pi i \alpha}$, satisfies mock Jacobi transformation properties (see [1, Proposition 3.2], and Theorem 2.1 below). We warn the reader that sometimes the symbol $Y^{+}$is also used to denote the (Jacobi) raising operator. In this paper, we adopt the notation from [1] in defining the function $Y^{+}$above. Given the quantum modularity of the one-variable specializations $\phi_{a / b}$, it is a natural question to investigate the Jacobi transformation properties of the two-variable function $Y^{+}$on a subset of $\mathbb{Q}^{2}$. It is also natural to seek explicit evaluations of the function $Y^{+}$. In this paper, we address both of these questions. To describe our results, we consider the following subset $\mathcal{Q}_{2}$ of $\mathbb{Q}^{2}$

$$
\mathcal{Q}_{2}:=\left\{\left(\frac{a}{b}, \frac{h}{k}\right) \in \mathbb{Q}^{2}: b, k \in \mathbb{N}, \operatorname{gcd}(a, b)=\operatorname{gcd}(h, k)=1, b \mid k\right\} .
$$

We also define the "error of modularity"

$$
H(z ; \tau):=\frac{i}{2} \frac{\vartheta(z ; \tau)}{\eta(\tau)} h(2 z ; \tau)-g(z ; \tau),
$$

where $\eta$ and $\vartheta$ are the modular and Jacobi forms, respectively, defined in (2.1), and the Mordell integrals $h$ and $g$ are given in (2.2). Our first result establishes the mock Jacobi and transformation properties of the two-variable combinatorial generating function $Y^{+}$.

Theorem 1.1. The following transformation properties hold.

(i) For $(z, \tau) \in(\mathbb{C} \times \mathbb{H}) \cup \mathcal{Q}_{2}$, we have that

$$
\begin{aligned}
& Y^{+}(z ; \tau)-e^{\frac{\pi i}{12}} Y^{+}(z ; \tau+1)=0, \\
& Y^{+}(z ; \tau)+i e^{\frac{3 \pi i z^{2}}{\tau}}(-i \tau)^{-\frac{1}{2}} Y^{+}\left(\frac{z}{\tau} ;-\frac{1}{\tau}\right)=-H(z ; \tau), \\
& Y^{+}(z ; \tau)+Y^{+}(z+1 ; \tau)=0, \\
& Y^{+}(z ; \tau)+e^{-6 \pi i z-3 \pi i \tau} Y^{+}(z+\tau ; \tau) \\
& \quad=e^{-5 \pi i z-\frac{25 \pi i \tau}{12}}\left(1-e^{4 \pi i z+2 \pi i \tau}\right)-\frac{i \vartheta(z ; \tau)}{\eta(\tau)}\left(e^{-2 \pi i z-\frac{\pi i \tau}{4}}-e^{-6 \pi i z-\frac{9 \pi i \tau}{4}}\right) .
\end{aligned}
$$

(ii) In particular, for $(z, \tau) \in \mathcal{Q}_{2}$, we have that

$$
\begin{aligned}
Y^{+}(z ; \tau)+i e^{\frac{3 \pi i z^{2}}{\tau}}(-i \tau)^{-\frac{1}{2}} Y^{+}\left(\frac{z}{\tau} ;-\frac{1}{\tau}\right) & =g(z ; \tau), \\
Y^{+}(z ; \tau)+e^{-6 \pi i z-3 \pi i \tau} Y^{+}(z+\tau ; \tau) & =e^{-5 \pi i z-\frac{25 \pi i \tau}{12}}\left(1-e^{4 \pi i z+2 \pi i \tau}\right) .
\end{aligned}
$$


The function $g$ on the right-hand side of (1.7) extends to a $C^{\infty}$ function on $(\mathbb{R} \backslash( \pm 1 / 6+\mathbb{Z})) \times \mathbb{R}^{\times}$, and the function on the right-hand-side of (1.8) extends to a $C^{\infty}$ function on $\mathbb{R}^{2}$.

Remarks.

(i) Theorem 1.1 shows that the function $Y^{+}$is a quantum Jacobi form of weight $1 / 2$ and index $-3 / 2$, a notion made more precise in Section 3.1. A direct calculation yields that $\mathcal{Q}_{2}$ is invariant under the action of the Jacobi group, so it is a natural "quantum Jacobi set."

(ii) The transformation in (1.4) was established previously by Rhoades and the authors [6] (see Section 2.1). For completeness, we have re-stated this result in Theorem 1.1.

It is not difficult to see, using its definition, that $Y^{+}$can be evaluated as a finite $q$-hypergeometric sum at $(z, \tau) \in \mathcal{Q}_{2}$ by truncating the infinite $q$-hypergeometric series in (1.1). Our next result provides a new, simpler expression for the strongly unimodal sequence rank generating functions $Y^{+}$as a two-variable Laurent polynomial, when evaluated at pairs of rationals in $\mathcal{Q}_{2}$. As we see below, the proof of Theorem 1.2 relies upon the quantum-Jacobi transformation properties on $\mathcal{Q}_{2}$ established in Theorem 1.1 (ii).

Theorem 1.2. Let $\tau=h / k$ and $z=a / b$ be such that $(z, \tau) \in \mathcal{Q}_{2}$. Then we have that

$$
Y^{+}(z ; \tau)=-\frac{1}{2} \zeta_{2 b}^{-5 a} \zeta_{24 k}^{-25 h} \sum_{j=0}^{k-1}(-1)^{j+1} \zeta_{2 k}^{-5 h j}\left(1-\zeta_{b}^{2 a} \zeta_{k}^{h(2 j+1)}\right) \zeta_{b}^{-3 j a} \zeta_{2 k}^{-3 j^{2} h} .
$$

As a corollary to Theorem 1.2, we obtain a new expression for radial limits involving the mock Jacobi partition rank function $R(w ; q)$ and the (meromorphic) Jacobi partition crank function $C(w ; q)$ (see Section 3.2 for their definitions), as originally studied by Ono, Rhoades, and the second author [6]. Namely if $w=\zeta_{b}^{a}$, as $q$ tends to another suitable root of unity $\zeta_{k}^{h}$ radially from within the unit disk, we have that

$$
\lim _{q \rightarrow \zeta_{k}^{h}}\left(R\left(\zeta_{b}^{a} ; q\right)-\zeta_{b^{2}}^{-a^{2} h^{\prime} k} C\left(\zeta_{b}^{a} ; q\right)\right)=-\left(1-\zeta_{b}^{a}\right)\left(1-\zeta_{b}^{-a}\right) U\left(\zeta_{b}^{a} ; \zeta_{k}^{h}\right)
$$

Here, $h^{\prime}$ is defined by $h h^{\prime} \equiv-1(\bmod k)$. This result of [6] in (1.9) generalizes and refines a more specific claim which pertains to the special case $\zeta_{b}^{a}=-1$, originally due to Ramanujan, and later proved by Watson [12]. From (1.9) and Theorem 1.2, we immediately obtain the following new evaluation of the radial limits (1.9).

Corollary 1.3. Let $1 \leq a<b, 1 \leq h<k$ with $\operatorname{gcd}(a, b)=\operatorname{gcd}(h, k)=1, b \mid k$ and $h^{\prime} \in \mathbb{Z}$ with $h h^{\prime} \equiv-1(\bmod k)$. Then, as $q \rightarrow \zeta_{k}^{\bar{h}}$ radially within the unit disc, we have that

$$
\begin{aligned}
\lim _{q \rightarrow \zeta_{k}^{h}}(R & \left.\left(\zeta_{b}^{a} ; q\right)-\zeta_{b^{2}}^{-a^{2} h^{\prime} k} C\left(\zeta_{b}^{a} ; q\right)\right) \\
& =\frac{1}{2}\left(1-\zeta_{b}^{-a}\right) \zeta_{b}^{-2 a} \zeta_{k}^{-h} \sum_{j=0}^{k-1}(-1)^{j+1} \zeta_{2 k}^{-5 h j}\left(1-\zeta_{b}^{2 a} \zeta_{k}^{h(2 j+1)}\right) \zeta_{b}^{-3 j a} \zeta_{2 k}^{-3 j^{2} h} .
\end{aligned}
$$

Alternative evaluations for $U(w ; q)$ (and hence $Y^{+}(z ; \tau)$ ) were also found in [5] for pairs of roots of unity $(w, q)=\left(\zeta_{b}^{a}, \zeta_{k}^{h}\right)$ with $b \mid k$, and also in [7] in the special case $(w, q)=\left(-1, \zeta_{k}^{h}\right)$ with $k$ even. In the latter special case $(w=-1)$, an alternative proof of results from $[6,7]$ may also be found in [16]. The methods of proof given in $[5,7,16]$ are different than those used here to establish Theorem 1.2. Moreover, the evaluations obtained in [5, 7] are $q$-hypergeometric in nature, again different from our polynomial evaluations.

The remainder of the paper is organized as follows: in Section 2 we review various properties of $U(w ; q)$, in Section 3 we recall properties of partition ranks and cranks and prove Theorem 1.1, and in Section 4 we establish Theorem 1.2. 


\section{ACKNOWLEDGEMENTS}

The authors thank Larry Rolen for fruitful conversations on related topics. Moreover they thank the anonymous referee for helpful comments.

\section{Properties of the unimodal Rank generating Function}

2.1. Modular transformations. In this section, we recall the modular Jacobi transformation properties of the normalized unimodal rank generating function $Y^{+}$on $\mathbb{C} \times \mathbb{H}$. To describe this, we require the function $H$ from (1.2), which is defined in terms of the modular and Jacobi forms (with $\left.q:=e^{2 \pi i \tau}\right)$

$$
\eta(\tau):=q^{\frac{1}{24}} \prod_{n=1}^{\infty}\left(1-q^{n}\right), \quad \vartheta(z ; \tau):=\sum_{n \in \frac{1}{2}+\mathbb{Z}} e^{2 \pi i n\left(z+\frac{1}{2}\right)} q^{\frac{n^{2}}{2}},
$$

and the Mordell integrals

$$
h(z ; \tau):=\int_{\mathbb{R}} \frac{e^{\pi i \tau t^{2}-2 \pi z t}}{\cosh (\pi t)} d t, \quad g(z ; \tau):=\frac{i}{\sqrt{3}} \int_{\mathbb{R}} e^{\frac{\pi i \tau t^{2}}{3}-2 \pi z t} \frac{\sinh \left(\frac{2 \pi t}{3}\right)}{\cosh (\pi t)} d t .
$$

Note that $\eta$ is a weight $1 / 2$ modular form (with multiplier) on $\mathrm{SL}_{2}(\mathbb{Z})$ wheras $\vartheta$ is a weight $1 / 2$, index $1 / 2$ Jacobi form. In particular, $\vartheta$ satisfies the following elliptic transformation law (where we have let $w:=e^{2 \pi i z}$, which we make use of below:

$$
\vartheta(z+\tau ; \tau)=-q^{-\frac{1}{2}} w^{-1} \vartheta(z ; \tau)
$$

In terms of the function $H$ in (1.2), we have the following Jacobi modular transformation properties; the transformation in (2.4) follows easily using the definition of $Y^{+}$, and the transformation in (2.5) was established in [1, Proposition 3.2].

Theorem 2.1. For $(z, \tau) \in \mathbb{C} \times \mathbb{H}$, we have that

$$
\begin{aligned}
Y^{+}(z ; \tau)-e^{\frac{\pi i}{12}} Y^{+}(z ; \tau+1) & =0 \\
Y^{+}(z ; \tau)+i e^{\frac{3 \pi i z^{2}}{\tau}}(-i \tau)^{-\frac{1}{2}} Y^{+}\left(\frac{z}{\tau} ;-\frac{1}{\tau}\right) & =-H(z ; \tau) .
\end{aligned}
$$

2.2. Quantum transformations. Quantum modular forms were originally introduced by Zagier [15] as follows.

Definition. A weight $k \in \frac{1}{2} \mathbb{Z}$ quantum modular form is a complex-valued function $f$ on $\mathbb{Q}$, such that for all $\gamma=\left(\begin{array}{ll}a & b \\ c & d\end{array}\right) \in \mathrm{SL}_{2}(\mathbb{Z})$, the functions $h_{\gamma}: \mathbb{Q} \backslash \gamma^{-1}(i \infty) \rightarrow \mathbb{C}$ defined by

$$
h_{\gamma}(x):=f(x)-\varepsilon(\gamma)(c x+d)^{-k} f\left(\frac{a x+b}{c x+d}\right)
$$

satisfy a "suitable" property of continuity or analyticity in (a subset of) $\mathbb{R}$.

Remark. We have modified Zagier's original definition in [15] to allow multipliers $\varepsilon(\gamma)$ in (2.6), i.e., suitable complex numbers such as those appearing in the theory of half-integral weight modular forms if $k \in \frac{1}{2} \mathbb{Z} \backslash \mathbb{Z}$. We may also allow quantum modular forms to transform on subgroups of $\mathrm{SL}_{2}(\mathbb{Z})$, and on appropriate subsets of $\mathbb{Q}$.

A particular quantum modular form is given by a special value of the unimodal rank generating function. Namely, Bryson, Ono, Pitman, and Rhodes [4] studied

$$
U(1 ; q)=\sum_{n=1}^{\infty} \sum_{m=-\infty}^{\infty}(-1)^{m} u(m, n) q^{n}=\sum_{n=1}^{\infty}\left(u_{e}(n)-u_{o}(n)\right) q^{n},
$$


where $u_{e}(n)$ (resp. $\left.u_{o}(n)\right)$ denotes the number of unimodal sequences of size $n$ with even (resp. odd) rank. They showed that for every root of unity $\zeta$,

$$
U(1 ; \zeta)=F\left(\zeta^{-1}\right)
$$

where Kontsevich's "strange" function is defined by

$$
F(q):=\sum_{n=0}^{\infty}(q ; q)_{n} .
$$

Prior to this, Zagier [13] proved that $F$ satisfies the "identity"

$$
F(q)=-\frac{1}{2} \sum_{n=1}^{\infty} n\left(\frac{12}{n}\right) q^{\frac{n^{2}-1}{24}},
$$

where $(\vdots)$ is the Kronecker symbol. Neither side of (2.8) makes sense simultaneously. Indeed, the right-hand side of (2.8) converges in the unit disk $|q|<1$, but nowhere on the unit circle. The identity (2.8) means that at roots of unity $\zeta, F(\zeta)$ (which is a finite sum) agrees with the limit as $q$ approaches $\zeta$ radially within the unit disk of the function on the right-hand side of (2.8). Moreover, Zagier proved that for $x \in \mathbb{Q}^{\times}$

$$
\phi(x)+(-i x)^{-\frac{3}{2}} \phi\left(-\frac{1}{x}\right)=\frac{\sqrt{3 i}}{2 \pi} \int_{0}^{i \infty} \frac{\eta(\rho)}{(\rho+x)^{\frac{3}{2}}} d \rho,
$$

where $\phi(x):=e^{-\frac{\pi i x}{12}} F\left(e^{-2 \pi i x}\right)$. Note that the constant $\sqrt{3 i} / 2 \pi$ in (2.9) is obtained explicitly in [4]. There, the authors also gave a new proof of $(2.9)$, using the fact that $U(1 ; q)$ is a (weak) mixed mock modular form for $|q|<1$.

By $[8,9]$, equation $(2.7)$ can also be interpreted in terms of quantum topology. In particular, based on the colored Jones polynomial for certain torus knots at roots of unity, the authors in $[8,9]$ introduced infinite families of quantum modular forms, including $F(q)$ and $U(1 ; q)$. In [5], the authors also generalized this picture to roots of unity other than $w=1$. That is, they defined a generalization $F(w ; q)$ of Kontsevich's function, with the property that $F(1 ; q)=F(q)$, and showed that $U\left(\zeta_{b}^{a} ; \zeta\right)=F\left(\zeta_{b}^{a} ; \zeta^{-1}\right)$, where $\zeta_{b}^{a}$ is any admissible root of unity (depending on $\zeta$ ), generalizing (2.7). The authors also produced quantum modular forms of weight $1 / 2$ from $F(w ; q)$, extending $(2.9)$.

\section{Proof of theorem 1.1 And QUANTUm Jacobi Forms}

3.1. Quantum Jacobi forms. We start by introducing the notion of quantum Jacobi forms.

Definition. A weight $k \in \frac{1}{2} \mathbb{Z}$ and index $m \in \frac{1}{2} \mathbb{Z}$ quantum Jacobi form is a complex valued function on $\mathbb{Q} \times \mathbb{Q}$ such that for all $\gamma=\left(\begin{array}{ll}a & b \\ c & d\end{array}\right) \in \mathrm{SL}_{2}(\mathbb{Z})$ and $(\lambda, \mu) \in \mathbb{Z}^{2}$ the functions

$$
\begin{aligned}
h_{\gamma}(z ; \tau) & :=\phi(z ; \tau)-\varepsilon_{1}(\gamma)(c \tau+d)^{-k} e^{-\frac{2 \pi i m c z^{2}}{c \tau+d}} \phi\left(\frac{z}{c \tau+d} ; \frac{a \tau+b}{c \tau+d}\right), \\
g_{(\lambda, \mu)}(z ; \tau) & :=\phi(z ; \tau)-\varepsilon_{2}((\lambda, \mu)) e^{2 \pi i m\left(\lambda^{2} \tau+2 \lambda z\right)} \phi(z+\lambda \tau+\mu ; \tau)
\end{aligned}
$$

satisfy a "suitable" property of continuity or analyticity in (a subset of) $\mathbb{R}^{2}$.

Remark. The $\varepsilon_{1}, \varepsilon_{2}$ are appropriate complex numbers, such as those appearing in the theory of halfintegral weight modular (Jacobi) forms. We may also allow quantum Jacobi forms to transform on subgroups of $\mathrm{SL}_{2}(\mathbb{Z})$, and on appropriate subsets of $\mathbb{Q} \times \mathbb{Q}$, which are in particular required to be closed under the action of the Jacobi group.

This definition is analogous to the definition of a quantum modular form in [15], just as the definition of a (holomorphic) Jacobi form is analogous to the definition of a (holomorphic) modular form. 
3.2. Partition ranks and cranks. In this section, we review the definitions of and basic properties of partition ranks and cranks. Both appear in (1.9) and Corollary 1.3, and ranks are used in our proof of Theorem 1.1 in Section 3.3.

The rank of an integer partition is defined to be its largest part minus the number of its parts. Partition cranks are given in terms of $o(\lambda)$, the number of ones in a partition $\lambda$, and $\mu(\lambda)$, the number of parts strictly larger than $o(\lambda)$. That is, the crank of a partition $\lambda$ is

$$
\operatorname{crank}(\lambda):= \begin{cases}\text { largest part of } \lambda & \text { if } o(\lambda)=0, \\ \mu(\lambda)-o(\lambda) & \text { if } o(\lambda)>0 .\end{cases}
$$

Let $N(m, n):=p(n \mid \operatorname{rank} \mathrm{m})$, and $M(m, n):=p(n \mid \operatorname{crank} \mathrm{m})$, where $p(n):=\#$ \{partitions of $n\}$. The well-known two-variable generating functions for partition ranks and cranks are

$$
\begin{aligned}
& R(w ; q):=\sum_{\substack{m \in \mathbb{Z} \\
n \geq 0}} N(m, n) w^{m} q^{n}=\sum_{n=0}^{\infty} \frac{q^{n^{2}}}{(w q ; q)_{n}\left(w^{-1} q ; q\right)_{n}}, \\
& C(w ; q):=\sum_{\substack{m \in \mathbb{Z} \\
n \geq 0}} M(m, n) w^{m} q^{n}=\prod_{n=1}^{\infty} \frac{1-q^{n}}{\left(1-w q^{n}\right)\left(1-w^{-1} q^{n}\right)},
\end{aligned}
$$

the first of which is a mock Jacobi form $[2,10,14]$, and the second of which is a meromorphic Jacobi form, using (3.1) below.

3.3. Proof of Theorem 1.1. In this section, we prove Theorem 1.1. We first point out that the transformation given in (1.5) follows easily from the definition of the function $Y^{+}$. Moreover, the Jacobi modular transformations (1.3) and (1.4) are given in Theorem 2.1. Thus, we are left to establish (1.6), (1.7), and (1.8), and the analytic properties claimed in Theorem 1.1.

For ease of notation, in this section we let $w:=e(z)$ and recall $q=e(\tau)$. From (1.4), the transformation in (1.7) follows, noting that by the Jacobi triple product identity

$$
\frac{\vartheta(z ; \tau)}{\eta(\tau)}=-i q^{\frac{1}{12}} w^{-\frac{1}{2}} \prod_{n=1}^{\infty}\left(1-w q^{n-1}\right)\left(1-w^{-1} q^{n}\right),
$$

so that if $(z, \tau) \in \mathcal{Q}_{2}$, then the second summand on the right-hand side of (1.4) vanishes.

To prove the elliptic transformations given in (1.6) and (1.8), we first decompose $U$ similarly as in the proof of [1, Lemma 3.1]. This gives that

$$
\begin{aligned}
& Y^{+}(z ; \tau)=w^{\frac{1}{2}} q^{-\frac{1}{24}} R^{*}(w ; q)+\frac{\vartheta(z ; \tau)}{\eta(\tau)} \mu(z,-z ; \tau), \quad \text { where } \\
& R^{*}(w ; q):=\frac{R(w ; q)}{1-w}, \quad \mu\left(z_{1}, z_{2} ; \tau\right):=\frac{e^{\pi i z_{1}}}{\vartheta\left(z_{2} ; \tau\right)} \sum_{n \in \mathbb{Z}} \frac{(-1)^{n} e^{2 \pi i n z_{1}} q^{\frac{n(n+1)}{2}}}{1-e^{2 \pi i z_{2}} q^{n}} .
\end{aligned}
$$

By $[3,(3.1)]$, we obtain

$$
w^{\frac{1}{2}} q^{-\frac{1}{24}} R^{*}(w ; q)=i w^{-1} q^{-\frac{1}{6}} \mu(3 z,-\tau ; 3 \tau)-i w q^{-\frac{1}{6}} \mu(3 z, \tau ; 3 \tau)-i \frac{\eta^{3}(3 \tau)}{\eta(\tau) \vartheta(3 z ; 3 \tau)} .
$$

We point out that due to work of Zwegers [17], the $\mu$-function is essentially a mock Jacobi form, upon suitable choices of parameters. In particular, we have the following elliptic transformation law from [17, Proposition $1.3(2)]$ :

$$
\begin{gathered}
\mu\left(z_{1}, z_{2} ; \tau\right)+e^{-2 \pi i\left(z_{1}-z_{2}\right)-\pi i \tau} \mu\left(z_{1}+\tau, z_{2} ; \tau\right)=-i e^{-\pi i\left(z_{1}-z_{2}\right)-\frac{\pi i \tau}{4}} . \\
6
\end{gathered}
$$


Thus

Similarly

$$
i w^{-1} q^{-\frac{7}{6}} \mu(3 z+3 \tau,-\tau ; 3 \tau)=-i w^{2} q^{\frac{4}{3}}\left(\mu(3 z,-\tau ; 3 \tau)+i w^{-\frac{3}{2}} q^{-\frac{7}{8}}\right)
$$

$$
-i w q^{\frac{5}{6}} \mu(3 z+3 \tau, \tau ; 3 \tau)=i w^{4} q^{\frac{4}{3}}\left(\mu(3 z, \tau ; 3 \tau)+i w^{-\frac{3}{2}} q^{\frac{1}{8}}\right) .
$$

Thus (using (2.3) for the final term in (3.3)), we obtain

$$
w^{\frac{1}{2}} q^{-\frac{1}{24}} R^{*}(w ; q)+w^{-3} q^{-\frac{3}{2}}(w q)^{\frac{1}{2}} q^{-\frac{1}{24}} R^{*}(w q ; q)=w^{-\frac{5}{2}} q^{-\frac{25}{24}}-w^{-\frac{1}{2}} q^{-\frac{1}{24}} .
$$

We next turn to the second summand in (3.2), using that $\mu\left(z_{1}+\tau, z_{2}+\tau ; \tau\right)=\mu\left(z_{1}, z_{2} ; \tau\right)[17$, Proposition 1.4 (4)], again (2.3), and twice (3.4), we obtain

$$
\vartheta(z+\tau ; \tau) \mu(z+\tau,-z-\tau ; \tau)=-w^{3} q^{\frac{3}{2}} \vartheta(z ; \tau)\left(\mu(z,-z ; \tau)+i w^{-1} q^{-\frac{1}{8}}-i w^{-3} q^{-\frac{9}{8}}\right) .
$$

Combining gives (1.6). The transformation (1.8) follows by again using (3.1).

It remains to show that the functions on the right-hand-sides of (1.7) and (1.8) are $C^{\infty}$ on $(\mathbb{R} \backslash( \pm 1 / 6+\mathbb{Z})) \times \mathbb{R}^{\times}$and $\mathbb{R}^{2}$, respectively. The result pertaining to (1.8) is clear. To show the claim for $g$ from (1.7), we re-write $g$ as

$$
g(z ; \tau)=\frac{1}{2 i \sqrt{3}} \sum_{ \pm} \pm h\left(z \pm \frac{1}{3} ; \frac{\tau}{3}\right)
$$

which was established in the proof of [1, Proposition 3.2]. We next restrict the domain in the variable $z$ by using that for $r \in \mathbb{Z}$, we have that

$$
h(z)+(-1)^{r+1} h(z+r)=\frac{2}{\sqrt{-i \tau}} \sum_{j=0}^{|r|-1}(-1)^{j} e^{\frac{\pi i}{\tau}\left(z+\operatorname{sgn}(r)\left(j+\frac{1}{2}\right)\right)^{2}},
$$

which is not hard to see by induction, using [17, Proposition 1.2 (1)] for $r=1$. Note that the right-hand side of (3.5) is analytic in $\mathbb{R} \times \mathbb{R}^{\times}$. Because we exclude $\pm 1 / 6+\mathbb{Z}$ in the statement of the theorem, and the function $z \mapsto h(z ; \tau)$ is even, it suffices to study it in $[0,1 / 2) \times \mathbb{R}^{\times}$. For this, we apply [17, Theorem $1.16(2)]$ with $a=0$ and $b=-z$, to re-write

$$
-h(z ; \tau)=\int_{0}^{i \infty} \frac{g_{\frac{1}{2},-z+\frac{1}{2}}(\rho)}{\sqrt{-i(\rho+\tau)}} d \rho, \quad \text { where } \quad g_{\alpha, \beta}(\tau):=\sum_{n \in \alpha+\mathbb{Z}} n e^{\pi i n^{2} \tau+2 \pi i n \beta} .
$$

We make the change of variables $\rho=i t$ and then split the integral in (3.6) into a sum of two integrals, one over the interval $(0,1)$ and one over $(1, \infty)$. In the first interval, we make the change of variables $t \mapsto 1 / t$ and use the modular inversion property from [17, Proposition 1.15 (5)]

$$
g_{\frac{1}{2},-z+\frac{1}{2}}\left(-\frac{1}{\tau}\right)=i e^{\pi i\left(-z+\frac{1}{2}\right)}(-i \tau)^{\frac{3}{2}} g_{-z+\frac{1}{2},-\frac{1}{2}}(\tau),
$$

which yields

$$
i h(z ; \tau)=\frac{i e^{\pi i\left(-z+\frac{1}{2}\right)}}{\sqrt{-i \tau}} \int_{1}^{\infty} \frac{g_{-z+\frac{1}{2},-\frac{1}{2}}(i t)}{\sqrt{t+\frac{i}{\tau}}} d t+\int_{1}^{\infty} \frac{g_{\frac{1}{2},-z+\frac{1}{2}}(i t)}{\sqrt{t-i \tau}} d t .
$$

Since we exclude $\tau=0$, we thus have to show that

$$
F(z, \tau):=\int_{1}^{\infty} \frac{g_{z_{1}, z_{2}}(i t)}{\sqrt{t-i \tau}} d t
$$


with $\left(z_{1}, z_{2}\right) \in\{(1 / 2,-z+1 / 2),(-z+1 / 2,-1 / 2)\}$ is $C^{\infty}$ in $[0,1 / 2] \times \mathbb{R}^{\times}$. For this, we establish the following bounds $(\ell \in \mathbb{N}, v:=\operatorname{Im}(\tau))$ using that the $n=0$ terms are the dominant terms

$$
\begin{aligned}
\left|g_{z_{1}, z_{2}}(\tau)\right| & \ll\left|z_{1}\right| e^{-\pi z_{1}^{2} v}, \\
\frac{\partial}{\partial z^{\ell}} g_{\frac{1}{2},-z+\frac{1}{2}}(\tau) & =(-2 \pi i)^{\ell} \sum_{n \in \mathbb{Z}}\left(n+\frac{1}{2}\right)^{\ell+1} e^{\pi i\left(n+\frac{1}{2}\right)^{2} \tau+2 \pi i\left(n+\frac{1}{2}\right)\left(-z+\frac{1}{2}\right)} \ll e^{-\pi\left(n+\frac{1}{2}\right)^{2} v}, \\
\frac{\partial}{\partial z^{\ell}} g_{-z+\frac{1}{2},-\frac{1}{2}}(\tau) & =\sum_{j=0}^{\ell+1} P_{j}(\tau) \sum_{n \in \mathbb{Z}}\left(n-z+\frac{1}{2}\right)^{j} e^{\pi i\left(n-z+\frac{1}{2}\right)^{2} \tau-\pi i\left(n-z+\frac{1}{2}\right)} \ll_{z}|\tau|^{\ell} e^{-\pi\left(z-\frac{1}{2}\right)^{2} v},
\end{aligned}
$$

where the $P_{j}$ are polynomials of degree at most $\ell$. This easily yields uniform bounds in $\tau$ and $z$, and we may apply the Leibnitz rule for indefinite integrals to prove the claim.

\section{Proof of Theorem 1.2}

We are now ready to prove Theorem 1.2. As in Section 3, we let $w=e(z)$ and $q=e(\tau)$.

Proof of Theorem 1.2. Suppose a function $f(z ; \tau)$ transforms in the elliptic variable as

$$
f(z+\tau ; \tau)=-w^{3} q^{\frac{3}{2}} f(z ; \tau)+w^{3} q^{\frac{3}{2}} r(z ; \tau)
$$

for some function $r(z ; \tau)$. By induction on $m \in \mathbb{N}_{0}$, it is not difficult to show that for all $m \in \mathbb{N}_{0}$

$$
\begin{aligned}
f(z+m \tau ; \tau) & =(-1)^{m} w^{3 m} q^{\frac{3}{2} m^{2}} f(z ; \tau)+\mathcal{G}_{m, r}(z ; \tau), \quad \text { where } \\
\mathcal{G}_{m, r}(z ; \tau) & :=(-1)^{m+1} \sum_{j=0}^{m-1}(-1)^{j} r(z+j \tau ; \tau) w^{3(m-j)} q^{\frac{3}{2}\left(m^{2}-j^{2}\right)} .
\end{aligned}
$$

By (1.8), the function $Y^{+}(z ; \tau)$ transforms for $(z, \tau) \in(a / b, h / k) \in \mathcal{Q}_{2}$ as in (4.1) with

$$
r(z ; \tau):=w^{-\frac{5}{2}} q^{-\frac{25}{24}}\left(1-w^{2} q\right) .
$$

Thus, from (4.2) with $m=k$, we have

$$
\left[Y^{+}(z+h ; \tau)\right]_{(z, \tau)=\left(\frac{a}{b}, \frac{h}{k}\right)}=\left[(-1)^{k} w^{3 k} q^{\frac{3}{2} k^{2}} Y^{+}(z ; \tau)+\mathcal{G}_{k, r}(z ; \tau)\right]_{(z, \tau)=\left(\frac{a}{b}, \frac{h}{k}\right)} .
$$

On the other hand, using (1.5), we have for any $h \in \mathbb{Z}$

$$
Y^{+}(z+h ; \tau)=(-1)^{h} Y^{+}(z ; \tau) \text {. }
$$

We obtain

$$
\left[\left((-1)^{h}-(-1)^{k} w^{3 k} q^{\frac{3}{2} k^{2}}\right) Y^{+}(z ; \tau)\right]_{(z, \tau)=\left(\frac{a}{b}, \frac{h}{k}\right)}=\left[\mathcal{G}_{k, r}(z ; \tau)\right]_{(z, \tau)=\left(\frac{a}{b}, \frac{h}{k}\right)} .
$$

The factor in front of $Y^{+}$in (4.3) evaluates as $2(-1)^{h}$, using the fact that if $h$ is even, then $k$ must be odd. This proves the theorem.

\section{REFERENCES}

[1] K. Bringmann, A. Folsom, R. Rhoades, Unimodal sequences and "strange" functions: a family of quantum modular forms, Pacific J. Math. 274 (2015), 1-25.

[2] K. Bringmann, K. Ono, Dyson's ranks and Maass forms, Ann. of Math. 171 (2010), 419-449.

[3] K. Bringmann, S. Zwegers, Rank-Crank type PDE's and non-holomorphic Jacobi forms, Math. Research Letters 17 (2010), 589-600.

[4] J. Bryson, K. Ono, S. Pitman, R. Rhoades, Unimodal sequences and quantum and mock modular forms, Proc. Natl. Acad. Sci. USA 109 (2012), 16063-16067.

[5] A. Folsom, C. Ki, Y. Truong Vu, B. Yang, Strange combinatorial quantum modular forms, submitted. 
[6] A. Folsom, K. Ono, R. Rhoades, Mock theta functions and quantum modular forms, Forum of Math. Pi 1 (2013), e2.

[7] A. Folsom, K. Ono, R. Rhoades, Ramanujan's radial limits, Ramanujan 125, 91-102, Contemp. Math. 627, Amer. Math. Soc., Providence, RI, 2014.

[8] K. Hikami, Difference equation of the colored Jones polynomial for torus knot, Int. J. Math. 15 (2004) 959-965.

[9] K. Hikami, J. Lovejoy, Torus knots and quantum modular forms, Research in the Mathematical Sciences 2.2 (2015), 1-15.

[10] S-Y Kang, Mock Jacobi forms in basic hypergeometric series, Compositio Math. 145 (2009), 553-565.

[11] R. Rhoades, Asymptotics for the number of strongly unimodal sequences, IMRN 3 (2014), 700-719.

[12] G. Watson, The final problem: An account of the mock theta functions, J. London Math. Soc. 2 (1936), 55-80.

[13] D. Zagier, Vassiliev invariants and a strange identity related to the Dedekind eta-function, Topology 40 (2001), 945-960.

[14] D. Zagier, Ramanujan's mock theta functions and their applications (after Zwegers and Ono-Bringmann), Astérisque 326 (2009) Séminaire Bourbaki. Vol. 2007/2008.

[15] D. Zagier, Quantum modular forms, Quanta of maths, 659-675, Clay Math. Proc., 11, Amer. Math. Soc., Providence, RI, 2010.

[16] W. Zudilin, On three theorems of Folsom, Ono and Rhoades, Proc. Amer. Math. Soc. 143 (2015), 1471-1476.

[17] S. Zwegers, Mock theta functions, Ph.D. Thesis, Universiteit Utrecht, 2002.

Mathematical Institute, University of Cologne, Weyertal 86-90, 50931 Cologne, Germany

E-mail address: kbringma@math.uni-koeln.de

Department of Mathematics and Statistics, Amherst College, Amherst, MA 01002, USA

E-mail address: afolsom@amherst.edu 